Case Report:

\title{
Intraoperative Neurophysiology Monitoring During Selective Dorsal Rhizotomy for Spastic Cerebral Palsy
}

\author{
Sina Salehi ${ }^{1}$ (D) Hamid Nemati ${ }^{1,2}$ (D) Ahmad Soltani $^{3 *}$ (D)
}

1. Shiraz Neuroscience Research Center, Shiraz University of Medical Sciences, Shiraz, Iran

2. Department of Neurology, School of Medicine, Shiraz University of Medical Sciences, Shiraz, Iran

3. Department of Neurosurgery, School of Medicine, Shiraz University of Medical Sciences, Shiraz, Iran

$\begin{aligned} & \text { Use your device to scan } \\ & \text { and read the article online }\end{aligned}$
Spastic Cerebral Palsy. Iran J Neurosurg. 2018; 4(2):117-122. http://dx.doi.org/10.32598/irjns.4.2.117
doi: : http://dx.doi.org/10.32598/irjns.4.2.117

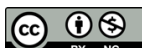

Funding: See Page 121

Article info:

Received: 13 October 2017

Accepted: 05 February 2018

Available Online: 01 April 2018

Keywords:

Intraoperative neurophysiology monitoring, Spastic cerebral palsy, Dorsal rhizotomy

\section{ABSTRACT}

Background and Importance: Selective Dorsal Rhizotomy (SDR) is a neurosurgical procedure currently used as a surgical treatment of children with spasticity in their legs. In SDR, the dorsal roots from L2 to S1 or S2 is partially sectioned. The primary goal of SDR is to reduce spasticity and improve the range of movement with preservation of muscle strength. The dorsal roots involved in spasticity are identified on the basis of intraoperative electrophysiological stimulation. Currently, SDR is commonly performed for the treatment of spastic cerebral palsy in children.

Case Presentation: We report an 8-year-old child with spastic cerebral palsy who underwent intraoperative neurophysiology monitoring during SDR for treating his spasticity. Before the operation, the patient's examination revealed more spasticity at the lower extremities, mild spasticity at the upper extremities, and occurrence of fixed contracture of his both ankle joints. Intraoperative neuromonitoring (consisting of motor evoked als, direct nerve root stimulation, and free run electromyography) was performed during the operation. Electrophysiological monitoring was initially used to help differentiate between the ventral and dorsal roots and cutting the abnormal sensory rootlets.

Conclusion: After the operation, his motor power of the lower extremities in the proximal and distal muscles was 4 out of 5 , his saddle sensation became normal, and there was no urinary and stool incontinency. Four weeks after the operation, he could walk about $10 \mathrm{~m}$ without help. His examination 2 and 4 weeks after the operation showed was no sign of sensory deficits, urinary, or stool incontinency. Two months after the operation, the patient could walk independently without help while before the operation, he could not.

* Corresponding Author: 


\section{Highlights}

- Selective dorsal rhizotomy (SDR) is a surgical treatment of children with spastic cerebral palsy.

- Intraoperative neuromonitoring improves SDR safety and outcomes.

- Although our case had fixed contracture of his both ankle joints, our treatment results were quite satisfactory.

\section{Plain Language Summary}

Cerebral palsy is one of the most common congenital neurological conditions in children. It is also one of the main causes of motor disability and spasticity in the childhood. Selective Dorsal Rhizotomy (SDR) is the most common neurosurgical procedure currently used for the treatment of children with spasticity in their legs. In SDR, some of the spinal cord dorsal roots are partially sectioned and cut. The primary goal of SDR is to reduce spasticity and improve the range of movement while preserving the muscle strength. We report an 8-year-old child with spastic cerebral palsy, who underwent Intraoperative Neurophysiology Monitoring (IONM) during SDR for treating his spasticity. Monitoring the state of the nervous system in "real-time" during surgery alerts surgeons of potential evolving neurologic injury thus improving safety and outcomes of SDR. To our knowledge, this is the first case in Iran who underwent IONM during SDR. When there was no IONM, the results could not be satisfactory because in this procedure the surgeon may miss cutting enough affected dorsal rootlets so some spasticity might remain in the patient. Two months after the operation, the patient could walk independently while before the operation, he could not.

\section{Introduction}

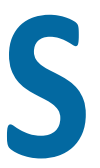

elective Dorsal Rhizotomy (SDR) is a neurosurgical procedure currently used as a surgical treatment of children with spasticity in their legs [1, 2]. Although the procedure has been successful in adolescents and even young adults, it is generally performed in 3- to 8-year-old children [2]. The term "selective" in SDR suggests an ability to select out the abnormally responsive dorsal rootlets, while preserving normally functioning ones. In SDR, partial sectioning of the dorsal roots from L2 to S1 or S2 is usually performed $[3,4]$.

The primary goal of SDR is to reduce spasticity and improve the range of movement with preservation of muscle strength by identifying that part of dorsal roots involved in spasticity on the basis of intraoperative electrophysiological stimulation $[2,5,6]$. The amount of sectioned rootlets is guided by clinical findings and IntraOperative Neurophysiology Monitoring (IONM) [6]. The purpose of IONM is to subdivide and section only portions of the dorsal root, thereby preserving sensory innervation and allowing motor retaining of the affected muscle group.

Spasticity is thought to be enhanced by sensory input onto the anterior horn cells that is unopposed by the descending inhibitory influences of the corticospinal tract. SDR is based on the hypothesis that reducing the degree of abnormal sensory input into the spinal reflex arc will result in diminished spasticity. This relative reduction of descending inhibitory impulses in the corticospinal pathways results in relative overactivity of the affected muscles (increased motor output), leading to increased muscle tone (i.e., spasticity). Intraoperative neuromonitoring uses continuous recording of Electromyography (EMG) in lower limb muscles, anal sphincter, and its response to simulation of selected rootlets $[2,6]$.

Intraoperative electrophysiological stimulation can be valuable in identification signs of spasticity and achieving a balance between elimination of spasticity and preservation of underlying strength $[2,6,7]$. EMG monitoring helps also avoid complications, especially sphincter paralysis and sensory loss in extremities [8] Patients who ultimately benefit most from the procedure typically have pure spasticity involving the lower limbs, good cognitive function, and strength, no fixed contractures and postural stability. We report herein a case with spastic Cerebral Palsy (CP) who underwent IONM during SDR for treating his spasticity. To the best of our knowledge this is the first case in Iran who underwent IONM during SDR.

\section{Case Presentation}

An 8-year-old child with a past history of prematurity, epilepsy, severe spasticity, and scissoring gait was admit- 
ted to Chamran Hospital, affiliated to Shiraz University of Medical Sciences, with chief complaint of spasticity. Until the age of 5, he was under antiepileptic drug medication (Sodium Valproate $20 \mathrm{mg} / \mathrm{kg}$ ). However, his antiepileptic drugs was later tapered and stopped because he had no epilepsy for 2 consecutive years. His cognitive functions were good as he could go to school and his learning abilities were similar to other children of the same age. His disease was not progressive until the age of 8 years. He had been undergone an adductor release surgery on his ankle to reduce dorsiflexion when he was 5 years old. The operation had reduced his scissoring gait, but his spasticity had not been significantly improved even by taking maximum dosage of antispastic drug (Baclofen 10 mg BID).

Before the operation, the patient could sit down without help and his muscle power was good, but he had some difficulties in walking without help due to the spasticity. The patient's examination before the operation revealed that he had more spasticity at the lower extremities, but lower spasticity at the upper extremities, and experienced fixed contracture at his ankle joints. In supine position, both of his lower extremities were flexed in both knees and hip joints. Other differential diagnoses such as neurodegenerative disorders were rolled out by the neurosurgeon in the examination and the patient who had spastic cerebral diplegia was planned to undergo SDR. Therefore, he was referred to a neurosurgeon to perform SDR to reduce his spasticity and improve gait.

The patient was placed prone and the sonography was used to confirm the level of conus medullaris. For the induction of anesthesia, intravenous propofol and remifentanil were administered without any medication for muscle relaxant to allow electrophysiological monitoring. After antiseptic painting, an 8-cm skin incision was made in the midline and laminectomy of T12 and partial
L1 was performed. The dura was opened longitudinally with the use of an intraoperative microscope. Intraoperative nerve monitoring, consisting of Motor Evoked Potentials (MEP), direct Nerve Root Stimulation (dNRS), and free run electromyography (fEMG), was performed during the operation. At the beginning and at the end of the operation, the MEP was performed to ensure of holding the motor power of the lower extremities. In order to do MEP, transcranial electrical stimulation was performed by inserting the subdermal electrodes at C3C4 scalp location and the MEP signals of 5 muscles, including right and left abductor halluces, tibialis anterior lateral gastrocnemius, semitendinosus, and vastus lateralis muscles were recorded. Direct nerve root stimulation was performed by electrical stimulation of ventral and dorsal nerve rootlets using $90^{\circ}$ bipolar hook electrodes with $1 \mathrm{~cm}$ between poles.

At first, L1 root was found at the left side from the respected neural foramen and was traced back to conus medullaris. Then, $\mathrm{L} 2$ root was detected at the conus. After meticulous arachnoid dissection and by considering any changes in electrophysiological signals, the dorsal root of L2 was safely separated from the ventral root so that a whitish cleft on the lateral side of the cord was clearly seen and a cotton patty was inserted between the dorsal and ventral root of $L 2$. In the same way, the roots from $L 2$ to S1 were dissected free from the ventral root and finally cotton patty was removed and replaced by small rubber sheet which separated the dorsal roots of $L 2$ to S2 from the rest of ventral roots and also from adjacent proximal and distal roots.

For confirming the visual differentiation between the ventral and dorsal roots, the roots were first stimulated by a single pulse of 1 to $3 \mathrm{~mA}$. If the single pulse stimulation of the root caused EMG response, the root was labeled as "ventral," otherwise it was labeled as "dorsal". Each dorsal root was divided into 3 to 4 rootlets and

Table 1. EMG responses for dorsal rhizotomy of spastic cerebral palsy

\begin{tabular}{cr}
\hline Grade & EMG Responses \\
\hline 0 & Unsustain or single discharge in response to train of stimulation \\
1 & Sustain discharges from muscles innervated through the segment stimulated in the ipsilateral lower extremity \\
2 & Sustain discharges from muscles innervated through the segment stimulated, as well as muscles innervated through the \\
3 & segment distant to the stimulated segment \\
4 & Sustain discharges from both ipsilateral and contralateral muscles
\end{tabular}


Table 2. The patient's lower limb spasticity index based on modified Ashworth scale for spasticity

\begin{tabular}{cccc}
\hline Item & Before the Operation & After the Operation & Comments \\
\hline Hip flexor & 3 & 0 & \\
\hline Hip extensor & $2-3$ & 0 & \\
\hline Hip abductor & $2-3$ & 0 & Previous adductor release \\
\hline Hip adductor & $2-3$ & 0 & \\
\hline Knee flexor & 3 & 1 & \\
\hline Knee extensor & $2-3$ & $1-0$ & Fixed contracture \\
\hline Ankle flexor & 4 & 3 & Fixed contracture \\
\hline Ankle extensor & 4 & 3 & \\
\hline
\end{tabular}

each rootlet was suspended by hook electrodes. Then, while recording the EMG from the muscles, the dorsal rootlets were stimulated with pulses of 1-3 mA with a frequency of $50 \mathrm{~Hz}$. EMG responses for dorsal rhizotomy of spastic CP were graded according to Table 1 (Adapted from Youmans Neurological Surgery, $7^{\text {th }}$ Edition) [9]. $s$
Figure 1 demonstrates an example of the rootlet stimulation during the operation with EMG responses of varying grade. Dorsal rootlets with EMG responses of grades 3 or more were cut and this procedure was performed bilaterally for all dorsal roots of L2 to S1. During the operation, we were careful to detect any

a.

b.

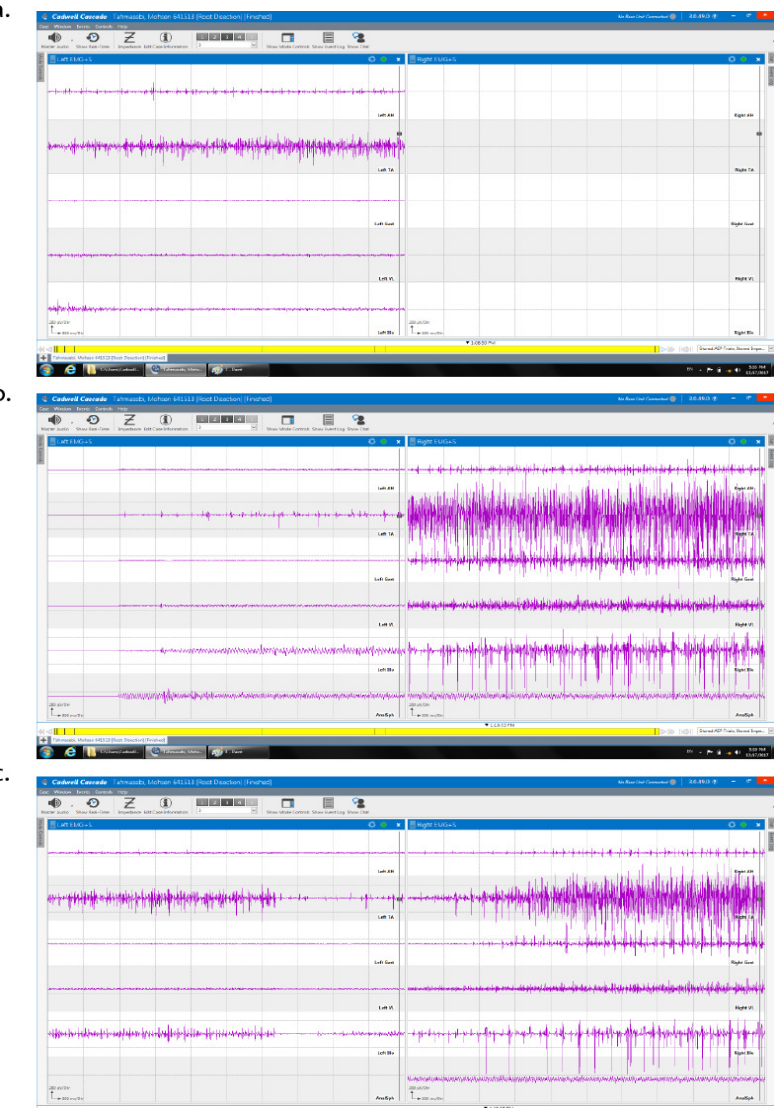

Figure 1. Dorsal rootlet stimulation during the operation with EMG responses of varying grades

Right and left panes show the EMG response of right and left muscles. EMG traces from upper to lower in each pane correspond to abductor halluces, tibialis anterior, lateral gastrocnemius, semitendinosus, and vastus lateralis muscles. (a) EMG response of grade 1. (b) EMG response of grade 3. (c) EMG response of grade 4. 
sustained EMG responses by manipulating around the conus medullaris. For three times, we had to wait about 5 minutes until EMG responses returned to normal before continuing the surgery.

Table 2 presents the degree of the spasticity before and after the operation measured by the modified Ashworth scale [10]. The patient had voluntary urinary excretion 72 hours after the operation and had no motor neurological deficits. After the operation, the patient was hospitalized for 1 day at our intensive care unit and 4 days later was discharged from the hospital. His motor power of the lower extremities in proximal and distal muscles was 4 out of 5 , his saddle sensation was normal, and there was no urinary and stool incontinency. While he was in a supine position, both of his lower extremities were extended in both knees and hip joints. $T$

Occupational therapy was begun at 2 weeks postoperatively. At 4 weeks after the operation, when the patient was revisited, he could walk about $10 \mathrm{~m}$ without help, his examination showed no sign of sensory deficits, urinary, or stool incontinency. Two months after the operation, the patient could walk independently without help while before the operation, he could not.

\section{Discussion}

To our knowledge, this is the first case in Iran who underwent IONM during SDR because dorsal rhizotomy in Iran has often been performed without IONM. When IONM is not available, the results cannot be satisfactory because the surgeon is unable to cut enough affected dorsal rootlets and some spasticity may remain. Comparable to other studies, a strong decrease was found in the spasticity of our patient, measured with the Ashworth scale (a widely used scale for clinical assessment) [11]. His results were quite satisfactory and he could walk independently and without help just two months after the operation.

Selective dorsal rhizotomy is usually performed via multilevel laminectomies to access each specific root in neural foramen, usually from L1 to S1. However, it increases the risk of late lumbar spinal instability and injury to the ventral roots. Another method is to perform laminectomy at a single level of the conus medullaris and separate posteriorly located dorsal roots that can be more easily separated from the ventral roots. It causes a significant reduction in the risk of the spinal instability, the duration of surgery, and injuries to the motor roots [12]. In our procedure, we performed the latter method and dissected the sensory roots at the conus medullaris that was a very sensitive procedure calling for enough experience and delicacy. Electrophysi- ological monitoring was used first to help differentiate between the ventral and dorsal roots and cutting the abnormal sensory rootlets.

\section{Conclusion}

IONM helps the surgeon to feel comfortable and more confident about the safety of the operation at hand. Otherwise speaking, surgeons can perform more high risk procedures with IONM. Selective dorsal rhizotomy with support of IONM can provide a permanent treatment for spasticity. It can be used in patients older than 2 years, but it is not recommended for patients younger than 2 years because spontaneous remission may occur when the patient is 1 year old $[13,14]$. Although our case was 8 years old when he underwent the operation and had also fixed contracture at his ankle joints, our results were quite satisfactory and was comparable to other studies [15]. After the operation, both of his lower extremities were extended in both knees and hip joints. It is worth to mention that if such patients undergo operation when they are about 2 to 3 years old and they will have no fixed contracture at their joints, the procedure can have the best therapeutic effect.

\section{Ethical Considerations}

Compliance with ethical guidelines

Surgical procedure and intraoperative neuromonitoring technique were explained to the patient's parents and written inform consent was obtained from them before the operation.

\section{Authors contribution}

The authors contributions are as follows: Conceptualization: Sina Salehi, Hamid Nemati; Methodology: All authors; Investigation: All author; Writing original draft: Sina Salehi, Ahmad Soltani; and Writing review and editing: Sina Salehi.

\section{Conflict of interest}

Authors declare that they have no conflict of interest.

\section{Funding}

This study is self-funded.

\section{Acknowledgements}

We would like to thank professor Majid Reza Farokhi for his valuable support to establish intraoperative neuromonitoring at Shiraz University of Medical Sciences. 


\section{References}

[1] Aquilina K, Graham D, Wimalasundera N. Selective dorsal rhizotomy: An old treatment re-emerging. Archives of Disease in Childhood. 2015 100(8):798-802. [DOI:10.1136/archdischild-2014-306874] [PMID]

[2] Farmer JP, McNeely PD. Surgery in the dorsal roots for children with cerebral palsy. Operative Techniques in Neurosurgery. 2005; 7(3):153-6. [DOI:10.1053/j.otns.2005.01.001]

[3] Trost JP, Schwartz MH, Krach LE, Dunn ME, Novacheck TF. Comprehensive short-term outcome assessment of selective dorsal rhizotomy. Developmental Medicine \& Child Neurology. 2008; 50(10):76571. [DOI:10.1111/j.1469-8749.2008.03031.x] [PMID]

[4] Nordmark E, Josenby AL, Lagergren J, Andersson G, Stromblad LG, Westbom L. Longterm outcomes five years after selective dorsal rhizotomy. BMC Pediatrics. 2008; 8:54. [DOI:10.1186/1471-2431-8-54] [PMID] [PMCID]

[5] Turner RP. Neurophysiologic intraoperative monitoring during selective dorsal rhizotomy. Journal of Clinical Neurophysiology. 2009; 26(2):82-4. [DOI:10.1097/WNP.0b013e31819f9077] [PMID]

[6] Steinbok P, Tidemann AJ, Miller S, Mortenson P, Bowen-Roberts T. Electrophysiologically guided versus non-electrophysiologically guided selective dorsal rhizotomy for spastic cerebral palsy: A comparison of outcomes. Child's Nervous System. 2009; 25(9):1091-6. [DOI:10.1007/s00381-009-0908-5] [PMID]

[7] Staudt LA, Nuwer MR, Peacock WJ. Intraoperative monitoring during selective posterior rhizotomy: Technique and patient outcome. Electroencephalography and Clinical Neurophysiology. 1995; 97(6):296309. [DOI:10.1016/0924-980X(95)00128-8]

[8] Deletis V, Vodusek DB, Abbott R, Epstein FJ, Turndorf H. Intraoperative monitoring of the dorsal sacral roots: Minimizing the risk of iatrogenic micturition disorders. Neurosurgery. 1992; 30(1):72-5. [DOI:10.1227/00006123-199201000-00013] [PMID]

[9] Winn HR. Youmans and Winn Neurological Surgery, 4-Volume Set (Youmans Neurological Surgery) $7^{\text {th }}$ edition. Amsterdam: Springer; 2016.

[10] Mutlu A, Livanelioglu A, Gunel MK. Reliability of Ashworth and Modified Ashworth scales in children with spastic cerebral palsy. BMC Musculoskeletal Disorders. 2008; 9:44. [DOI:10.1186/1471 2474-9-44] [PMID] [PMCID]

[11] McLaughlin JF, Bjornson K, Temkin N, Steinbok P, Wright V, Reiner A et al. Selective dorsal rhizotomy: Meta-analysis of three randomized controlled trials. Developmental Medicine \& Child Neurology. 2002; 44(1):17-25. [DOI:10.1017/S0012162201001608] [PMID]

[12] Graham D, Aquilina K, Cawker S, Paget S, Wimalasundera N. Singlelevel selective dorsal rhizotomy for spastic cerebral palsy. Journal of Spine Surgery. 2016; 2(3):195-201. [DOI:10.21037/jss.2016.08.08] [PMID] [PMCID]

[13] Arens LJ, Peacock WJ, Peter J. Selective posterior rhizotomy: A longterm follow-up study. Child's Nervous System. 1989; 5(3):148-52. [DOI:10.1007/BF00272116] [PMID]

[14] Nelson KB, Ellenberg JH. Children who 'outgrew' cerebral palsy. Pediatrics. 1982; 69(5):529-36. [PMID]

[15] Van Schie PE, Vermeulen RJ, van Ouwerkerk WJ, Kwakkel G, Becher $J G$. Selective dorsal rhizotomy in cerebral palsy to improve functional abilities: Evaluation of criteria for selection. Child's Nervous System. 2005; 21(6):451-7. [DOI:10.1007/s00381-004-1105-1] [PMID] 